\title{
Importance of neurological assessment before bone marrow transplantation for osteopetrosis
}

\author{
M Abinun, T Newson, P W Rowe, T J Flood, A J Cant
}

\begin{abstract}
Neurological complications of malignant infantile osteopetrosis are well recognised; successful bone marrow transplantation, when performed early in life, can prevent or halt some of them. In a subgroup of infants osteopetrosis is associated with primary retinal degeneration and/or generalised neurodegeneration. Bone marrow transplantation, in spite of being successful in correcting the osseous and haematological abnormalities, does not influence the progressive course of the neurodegenerative disorder. Thus, the recognition of this subgroup of infants with a very poor prognosis is essential before deciding on bone marrow transplantation.

(Arch Dis Child 1999;80:273-274)
\end{abstract}

Keywords: osteopetrosis; retinal degeneration; generalised neurodegeneration; bone marrow transplantation

Department of

Paediatrics, Newcastle

General Hospital, The

Newcastle Upon Tyne

Hospitals NHS Trust,

Newcastle Upon Tyne

NE4 6BE, UK

M Abinun

T Newson

P W Rowe

T J Flood

A J Cant

Correspondence to:

Dr M Abinun, Children's

Bone Marrow

Transplantation Unit,

Newcastle General Hospital,

Westgate Road, Newcastle

Upon Tyne NE4 6BE, UK.

email: Mario.Abinun@

newcastle.ac.uk

Accepted 1 November 1998 haematological abnormalities and can also halt or prevent the neurological complications caused by nerve entrapment. ${ }^{2}$ However, in some children primary retinal degeneration (either isolated or as part of a generalised neurodegenerative disorder), rather than compression of the optic nerve, has been described as a cause of blindness. ${ }^{134}$ Because it has been shown that successful BMT has no effect on the progressive course of the neurodegenerative disorder associated with infantile osteopetrosis, ${ }^{15}$ careful neurological evaluation of children referred for BMT is essential before reaching a decision about this procedure.

\section{Patients}

Five infants with infantile osteopetrosis were referred to us for BMT between 1992 and 1996. All had radiographically demonstrated generalised osteosclerosis, anaemia, hepatosplenomegaly, and normal renal tubular function. Bone marrow examination revealed overproliferation of osteoclasts in three patients, but virtually no osteoclasts were present in two patients (patients 4 and 5). Patient 3 had undergone bilateral surgical optic nerve decompressions before being referred to us. Their presentations varied and included hypocalcaemic convulsions (patients 1 and 5), recurrent upper respiratory infections (patients 2,3 , and 5), and lethargy and poor feeding (patients 4 and 5). Table 1 shows the results of the detailed neurological assessments.

Patient 4, a male infant of consanguineous parents, had severe visual impairment with optic atrophy, abnormal retinal pigmentary changes, absent visually evoked responses, hypertonicity, microcephaly, and marked cerebral atrophy. Because of the early onset of severe neurological impairment, his

Table 1 Neurological assessments of patients

\begin{tabular}{|c|c|c|c|c|c|}
\hline & Patient 1 & Patient 2 & Patient 3 & Patient 4 & Patient 5 \\
\hline Age at diagnosis & 3 weeks & 5.5 months & 6 weeks & 2 weeks & 3 months \\
\hline Age at referral & 4 months & 6 months & 9 months & 7 weeks & 5 months \\
\hline Sex & Female & Male & Male & Male & Male \\
\hline \multicolumn{6}{|c|}{ Neurological findings } \\
\hline OFC (centile) & 75 & 90 & 3 & $<3$ & $>90$ \\
\hline Length (centile) & 25 & 50 & 3 & 3 & 9 \\
\hline Features & Normal & Delay in head control & Mild motor delay & Spastic tetraplegia & Developmental delay \\
\hline \multicolumn{6}{|l|}{ Brain imaging } \\
\hline CT & Normal & Ventricular dilatation & Normal & Marked cerebral atrophy & Mild cerebral atrophy \\
\hline MRI & NA & NA & NA & Cerebral atrophy & NA \\
\hline \multicolumn{6}{|c|}{ Ophthalmological findings } \\
\hline Clinical & Normal & Normal & Roving nystagmus & Roving nystagmus & No eye contact/"blind" \\
\hline Fundoscopy & Normal & Normal & Optic atrophy & $\begin{array}{l}\text { Optic atrophy, retinal } \\
\text { depigmentation }\end{array}$ & Normal \\
\hline VER & Normal & Normal & Delayed/attenuated & Absent & Delayed/ broadened \\
\hline ERG & Normal & Normal & Normal & NA & Normal \\
\hline \multicolumn{6}{|l|}{ ENT findings } \\
\hline AER & Normal & Normal & Absent & Normal & Normal \\
\hline
\end{tabular}

AER, auditory evoked response; CT, computed tomography; ENT, ear, nose and throat; ERG, electroretinogram; MRI, magnetic resonance imaging; NA, not available; OFC, occipitofrontal circumference; VER, visually evoked response. 
investigation was broadened to include neurodegenerative disorders, but his electroencephalogram, white cell lysosomal enzyme assays, and electron microscopy of skin biopsy showed no abnormalities. Our joint decision was that BMT was not indicated for this infant and he was discharged to his referring hospital with suggestions of palliative treatment with limited calcium intake and corticosteroids. Patient 5 had signs of developmental delay with visual impairment but normal hearing and his brain computed tomography results showed mild cerebral cortical atrophy. His fundi appeared normal, his visually evoked potential showed delayed and broadened flash responses, and his electroretinogram showed a normal response under photopic conditions but an attenuated response under scotopic conditions, possibly as a result of the less than ideal light exclusion at the time. After detailed evaluation, the joint opinion was against the diagnosis of neurodegenerative disorder and the patient underwent BMT, as did the first three patients from our series. Although successfully engrafted, unfortunately, patient 5 died on day 22 after transplantation of progressive veno-occlusive disease of the liver. His postmortem examination, including neurohistology, showed no evidence of lysosomal storage disorder.

\section{Discussion}

Generalised neurological impairment has been recognised in children with osteopetrosis and can vary from mild motor delay, often with macrocephaly and mild ventricular dilatation, to severe psychomotor retardation. ${ }^{1}$ Sensorineural deafness can occur owing to bony compression of the VIII nerve; however, conductive deafness has been reported more commonly either as a result of sclerosis of the middle ear ossicles or poor drainage of the eustachian tube. ${ }^{6}$ The commonest and often earliest neurological manifestation of infantile osteopetrosis is visual impairment, which is present in $75 \%$ of patients by the 1 st year of life. ${ }^{1}$ In the largest reported series of patients, $87 \%$ had irreversible visual impairment despite successful BMT and bone clearing; this indicates the need for BMT as soon as possible after diagnosis, preferably before 3 months of age. ${ }^{12}$ Surgical decompression of the optic nerves has been shown to improve visual function in some patients; however, this procedure should not delay BMT. ${ }^{2}$ Although the visual loss has usually been attributed solely to optic atrophy secondary to bony compression of the optic nerve, it can be caused by primary retinal degenera- tion, either isolated or as a part of a progressive generalised neurodegenerative disorder. ${ }^{1{ }^{34}}$ In some children, the association of osteopetrosis with neuronal ceroid lipofuscinosis has been documented, ${ }^{4}$ and in others osteopetrosis was associated with infantile neuroaxonal dystrophy. ${ }^{3}$

The form of infantile osteopetrosis associated with retinal or generalised neurodegeneration characterises a separate disease entity with a very poor prognosis. ${ }^{2}$ A report of two such patients who died, after successful BMT, of progressive neurodegenerative disease emphasises the importance of recognition of this disorder, ${ }^{25}$ and two of our patients (patients 4 and 5) demonstrated the need for very careful and detailed evaluation before the decision about BMT is made. To determine the cause of visual loss, even in the presence of optic atrophy, full ophthalmological assessment including electrophysiological testing (measurement of visually evoked responses and electroretinography) is required. ${ }^{7}$ Cranial imaging is mandatory, preferably with magnetic resonance scanning because it is better than computed tomography at detecting white matter degeneration. ${ }^{6}$ Tests to exclude known neuronal storage disorders (vacuolated lymphocytes on the blood film, white cell enzyme assays, skin or rectal biopsy for electron microscopy and histochemical analysis, and electroencephalography) should be considered if children present early and with major neurological abnormalities. A paediatric neurologist, ophthalmologist, and otorhinolaryngologist should be involved in the clinical evaluation of these patients.

We thank Dr D Gardner-Medwin, Mr MP Clarke, Dr K Mitchell, Dr IS Schofield, Mr D Miekle, Dr MM Reed, and Dr C Wright for their help in assessing and evaluating these patients.

1 Gerritsen EJA, Vossen JM, van Loo IHG, et al. Autosomal recessive osteopetrosis: variability of findings at diagnosis

2 Gerritsen EJA, Vossen JM, Fasth A, et al. Bone marrow transplantation for autosomal recessive osteopetrosis. A report from the working party on inborn errors of the European bone marrow transplantation group. $\mathcal{F}$ Pediatr 1994;125:896-902.

3 Rees H, Ang L-C, Casey R, George DH. Association of infantile neuroaxonal dystrophy and osteopetrosis: a rare autosomal recessive disorder. Pediatr Neurosurg 1995;22: $321-7$

4 Alroy J, Castognaro M, Skutelsky E, Lomakina I. Lectin histochemistry of infantile lysosomal storage disease
associated with osteopetrosis. Acta Neuropathol 1994;87: 594-7.

5 Solh H, Da Cunha AM, Giri N, et al. Bone marrow transplantation for infantile malignant osteopetrosis. $\mathcal{F}$ Pediatr Hematol Oncol 1995;17:350-5.

6 Elster AD, Theros EG, Key LL, Chen MYM. Cranial imaging in autosomal recessive osteopetrosis. Part II. Skull base ing in autosomal recessive osteopetrosis.

7 Thompson DA, Kriss A, Taylor D, et al. Early VEP and ERG evidence of visual dysfunction in autosomal recessive osteopetrosis. Neuropediatrics 1998;29:137-44. 